\title{
Dual Cherenkov and Scintillation Response to High-Energy Electrons of Rare-Earth-Doped Silica Fibers
}

\author{
Francesca Cova, ${ }^{1}$ Marco T. Lucchini, $, 2,3$ Kristof Pauwels, ${ }^{4,3, \dagger}$ Etiennette Auffray, ${ }^{3}$ Norberto Chiodini, ${ }^{1}$ \\ Mauro Fasoli, ${ }^{1}$ and Anna Vedda ${ }^{1}$ \\ ${ }^{1}$ Department of Materials Science, University of Milano-Bicocca, Via Cozzi 55, 20125 Milan, Italy \\ ${ }^{2}$ Princeton University, Princeton, New Jersey 08544, USA \\ ${ }^{3}$ EP-CMX Department, CERN, 1211 Geneva 23, Switzerland \\ ${ }^{4}$ Department of Physics, University of Milano-Bicocca, Piazza della Scienza 3, 20125 Milan, Italy
}

(Received 19 October 2018; revised manuscript received 8 January 2019; published 14 February 2019)

\begin{abstract}
The investigation of the characteristic luminescent response of Ce-doped silica fibers exposed to electrons in the $20-200-\mathrm{GeV}$ energy range is reported in this work to explore the feasibility of using silica-based fibers for a simultaneous dual-readout approach. The sol-gel method allows the preparation of either doped or undoped fibers with high aspect ratio and high purity, providing good flexibility and spatial resolution for the realization of a dual-readout detector. The dual Cherenkov and scintillation light emitted by silica-based fibers potentially offers applications in high-energy-physics calorimetry as well as in other fields, such as radiation monitoring in medicine, security, and industrial control. The response of the fibers, embedded in a tungsten-copper absorber block to obtain a spaghetti-like geometry in a high-energyphysics environment, is investigated through a test-beam campaign at the CERN Super Proton Synchrotron facility. The discrimination of Cherenkov and scintillation light is demonstrated and discussed, along with a detailed investigation of the scintillation properties of the material: time-resolved spectroscopy, relative light output, and attenuation length are evaluated. The results presented in this study can pave the way for further material engineering and future applications.
\end{abstract}

DOI: 10.1103/PhysRevApplied.11.024036

\section{INTRODUCTION}

Current and future ionizing-radiation detectors need novel materials and concepts to improve and optimize their performances. The idea of fiber-shaped scintillation sensors has recently been developed: their application perspectives include devices for real-time dosimetry in medical systems [1], devices for beam monitoring and tracking [2], and high-granularity calorimeters for highenergy-physics (HEP) experiments [3-6]. On the basis of the possibility of growing a great variety of fiber shapes and lengths, several calorimeter designs have been proposed [7], exploiting the flexibility of fibers to adapt for various needs. Fibers can be applied either as wavelength shifters for the collection and transport of light [8] or

\footnotetext{
*marco.toliman.lucchini@cern.ch

${ }^{\dagger}$ Currently at European Synchrotron Radiation Facility, 71 Avenue des Martyrs, 38043 Grenoble, France.

Published by the American Physical Society under the terms of the Creative Commons Attribution 4.0 International license. Further distribution of this work must maintain attribution to the author(s) and the published article's title, journal citation, and DOI.
}

as scintillators and Cherenkov radiators in sampling and dual-readout calorimeters. Fiber technology can allow a cost-effective implementation of the dual-readout approach to HEP measurements. Moreover, fibers enable the design of detectors with high spatial resolution, good timing properties, and radiation tolerance, which are essential requirements for future calorimeters [9].

Dual-readout calorimetry was proposed as a technique to determine the electromagnetic shower fraction for individual events, by comparison on an event-by-event basis of the signals generated in the form of Cherenkov and scintillation light [10]. It has recently been considered to improve the performance of hadronic calorimeters in future collider experiments (i.e., for the forthcoming high-luminosity phase of the Large Hadron Collider at CERN) [11]. Scintillation light is proportional to the total energy deposited by the electromagnetic shower particles, while Cherenkov light is produced only by charged, relativistic particles, which are almost exclusively found in the electromagnetic shower component. Several approaches were suggested for the dual-readout concept: among them, either the use of two independent active media or the a posteriori decomposition of scintillation and Cherenkov components of the light emitted by a single material $[12,13]$. 
Besides HEP experiments, dual readout is involved in several other fields. Dual Cherenkov and scintillation readout has also been proposed for calibration and monitoring of high-energy-electron-beam profiles in radiotherapy and for the optical imaging of ionizing radiation in the clinical field [14,15]; in addition, it can be used for applications in security, industrial x-ray imaging, and radiography $[16,17]$. Furthermore, dual readout can be exploited to probe the transport of energetic solar particles, allowing coverage of the energy range from tens to hundreds of $\mathrm{MeV}$ with a single detector [18].

Research on fibers based on inorganic crystalline scintillators has seen significant development in recent years, and their use with rare-earth (RE) ions as luminescent activators for high-energy-radiation detection has already been intensively studied: $\mathrm{Ce}: \mathrm{Y}_{3} \mathrm{Al}_{5} \mathrm{O}_{12}$ (YAG) single-crystal fibers $[19,20]$ and $\mathrm{Ce}: \mathrm{Lu}_{3} \mathrm{Al}_{5} \mathrm{O}_{12}(\mathrm{LuAG})$ fibers grown by the micro-pulling down technique $[21,22]$ have been characterized with beam tests and under high levels of $\gamma$ and proton irradiation.

Moreover, scintillators based on glass matrices could be a good alternative to heavy crystals, because of their simpler preparation procedure, better shaping possibilities, faster pulling rates, and lower costs of production [23-26]. The sol-gel technique was proven to allow good control, at a relatively low densification temperature, of incorporation of $\mathrm{RE}$ ions and of their dispersion inside a glass matrix [27,28]: the glass synthesis can be performed with use of high-purity precursors, reducing the level of unwanted impurities, which is an essential feature for the radiation hardness of such materials. Several studies were devoted to the realization of RE-doped silica glasses prepared by the sol-gel route for HEP applications [29,30]. Previous results proved that sol-gel silica-based fibers can be considered a good scintillator material with a suitable attenuation length: however, their radiation hardness has still to be optimized for applications in HEP experiments with very high levels of radiation [31].

In this work, we present an in-depth investigation of the possibility and the potentiality to use RE-doped silica fibers for the simultaneous Cherenkov and scintillation dual-readout approach. They are proposed as the active material in a spaghetti-like electromagnetic calorimeter (SpaCal) prototype; their scintillation properties, as the results of a test with electron beams in the energy range from 20 to $200 \mathrm{GeV}$ performed at the $\mathrm{H} 4$ beam line of the CERN Super Proton Synchrotron (SPS) North Area facility, are also reported.

\section{EXPERIMENTAL SETUP AND PROCEDURE}

\section{A. Sol-gel silica fibers and SpaCal module}

Ce-doped silica glasses are prepared by the solgel method with tetramethylorthosilicate (TMOS) and cerium(III) nitrate as precursors. Alcogels are formed after gelation and subsequently dried in a thermostatic chamber for a few weeks. The xerogels obtained are densified at $1225^{\circ} \mathrm{C}$ in an oxidizing $\left(\mathrm{O}_{2}\right)$ atmosphere to produce Cedoped preforms. Cylindrical fibers are then drawn with a fluorinated $\mathrm{SiO}_{2}$ cladding wrapping the RE-doped core, to guarantee light guiding by the core-cladding interface: the core diameter is $0.60 \mathrm{~mm}$ and the total fiber diameter is $0.75 \mathrm{~mm}$. The numerical aperture of these fibers is estimated to be approximately 0.17 . Fibers are left uncoated and are cut into 200-mm-long pieces.

$\mathrm{A} \mathrm{W}-\mathrm{Cu}(75 \%: 25 \%)$ absorber block of dimensions $60 \times$ $60 \times 200 \mathrm{~mm}^{3}$ made of square $1.1 \times 1.1 \mathrm{~mm}^{2}$ holes, 1.8 $\mathrm{mm}$ apart, is filled with fibers to obtain a sampling SpaCal prototype, shown in Fig. 1(b), in which the fibers are used as the active scintillating material. These dimensions and fiber packing are optimized according to practical constraints.

Five distinct channels of fibers in the SpaCal module are sketched in Fig. 1(a) and filled with 80 fibers each, for a total bundle diameter of approximately $15 \mathrm{~mm}$ : the fibers are Ce:YAG (central channel), silica doped with $0.002 \%$ Ce (top left), Kuraray SCSF-3HF plastic used as a reference (top right), silica doped with $0.05 \% \mathrm{Ce}$ (bottom left), and silica doped with $0.0125 \%$ Ce (bottom right).

We decided to focus the investigation on the response of Ce-doped silica fibers with the highest Ce concentration compared with that of plastic fibers.

A set of six Hamamatsu Photonics R5380 photomultiplier tubes (PMTs) with bialkali photocathodes and borosilicate glass windows of $20-\mathrm{mm}$ diameter are used to readout the light from the fibers in the SpaCal module: the top channels are read out only from the rear side and the bottom channels are read out from both sides of the fibers to exploit the use and the advantages of the double-side readout technique [21,31]. A silicon-rubber optical interface (EJ-560, Eljen Technology) with refractive index of 1.43 is used to couple the fibers and the PMT window to improve light extraction.

The module is mounted inside a light tight aluminum box providing optical and thermal insulation, with a watercooled system to maintain a constant temperature of $18 \pm 0.5^{\circ} \mathrm{C}$ : the box is installed on a remotely controlled horizontal-vertical $(x-y)$ table with a displacement range of $\pm 300 \mathrm{~mm}$ and positioning accuracy of approximately 1 $\mathrm{mm}$. The frame of reference used throughout this paper has the $x$ axis horizontal on the experimental table, the $y$ axis pointing vertically upward, and the $z$ axis aligned with and pointing in the same direction as the beam line.

The module is exposed to the beam in two different configurations, described in Fig. 1(c), hereafter referred to as "pointing" and "transverse." In the former case, the beam is aligned with the fibers; the secondary particles, produced during the primary interaction of the high-energy electrons impacting on the absorber and denoted as "shower," developed all along the fiber length, while laterally extending 


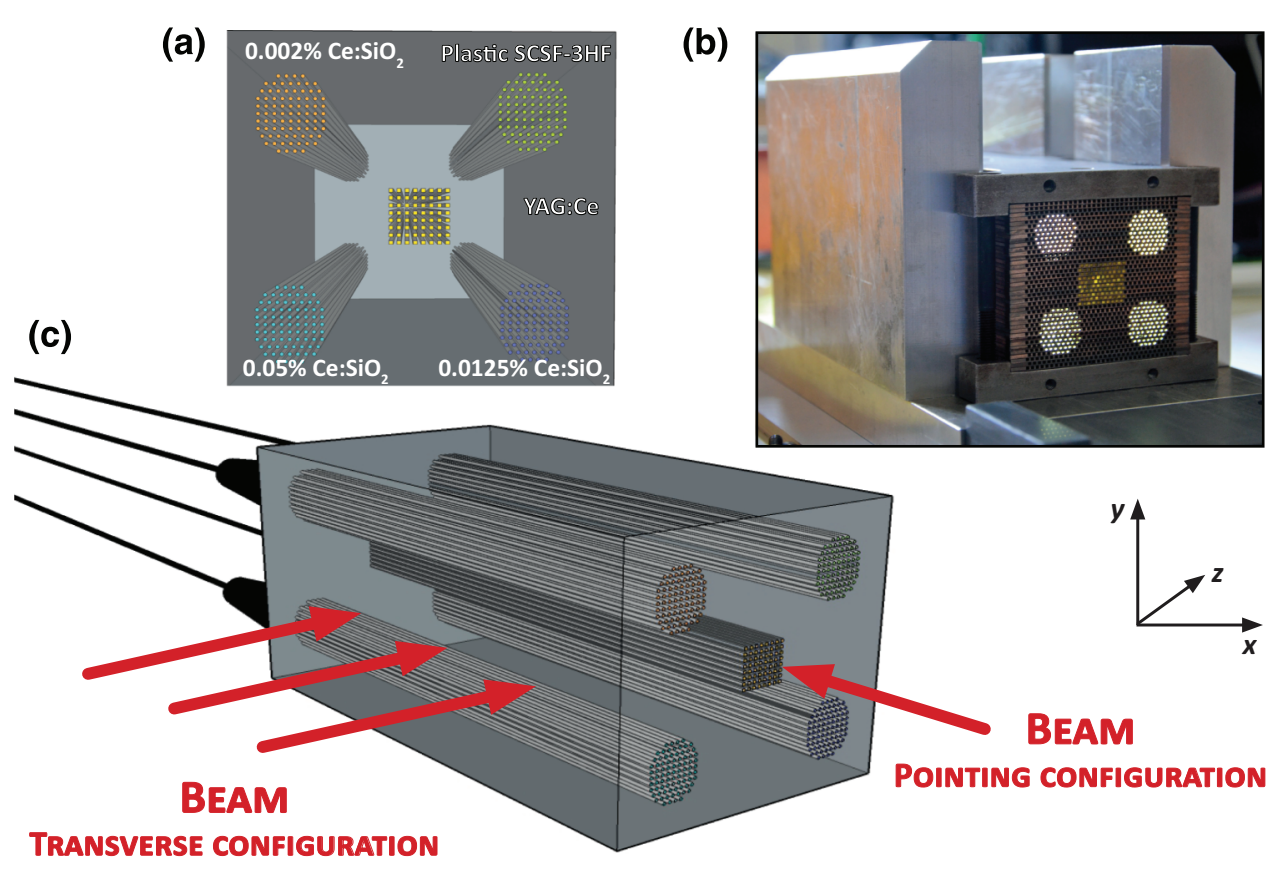

FIG. 1. SpaCal setup. (a) Front view of the SpaCal prototype: the labels describe the type of fibers in each channel. (b) Photograph of the SpaCal prototype made of five channels with 80 fibers each in a tungsten-copper absorber. (c) Side view of the SpaCal module, showing the pointing and transverse configurations.

inside the fiber bundle. The transverse dimension containing on average $90 \%$ of the shower's energy deposition (Molière radius [32]) is estimated to be approximately 17 $\mathrm{mm}$. In this configuration, the analysis of the time decay of the emitted light intensity (denoted as "pulse shape") and the study of the response of the fibers as a function of the varying incident-beam energy are performed. In the transverse configuration, the beam is impinging at a cross angle with respect to the fibers in order to analyze the attenuation profiles through a position scan, moving the table in discrete steps of approximately $30 \mathrm{~mm}$.

\section{B. Beam line}

The experiments are performed at the $\mathrm{H} 4$ beam line of the CERN SPS North Area facility, which provides electron beams in the $20-200-\mathrm{GeV}$ range with hadron and muon contamination below $0.2 \%$. Primary protons injected into the SPS interact with the T2 beryllium target to generate secondary particles, including electrons and pions. The type and energy of the particles are selected after the target by a set of magnets and collimators. The incoming electrons are detected by a set of four plastic scintillation counters used to trigger the data-acquisition system. The beam is composed of subsequent electron packets lasting $4 \mathrm{~s}$ and separated by around $30 \mathrm{~s}$. Each packet contains approximately $10^{4}$ electrons, among which approximately $10^{3}$ are useful for the measurements exploiting the coincidence with plastic scintillation counters. The impact point of the beam particles on the module is accurately measured with two sets of beam hodoscopes, shown in Fig. 2. Each set comprises two orthogonal planes of 64 scintillating fibers with square cross section of $0.5 \times 0.5 \mathrm{~mm}^{2}$, read out by a multianode PMT. Each plane provides, respectively, a measurement of the electron position in the $x$ direction and the $y$ direction with a nominal precision of less than $200 \mu \mathrm{m}[33]$.

\section{Experimental procedure}

The coincidence of signals from the beam counters is used to generate the trigger for the event acquisition, while the hodoscopes allow reconstruction of the impact point of the electrons on the SpaCal module. The signals from the PMTs are digitized by a CAEN V1742 module operating at a sampling speed of $2.5 \mathrm{GHz}$ and providing a time gate for the recorded pulses of $350 \mathrm{~ns}$. The response of a particular channel varies with the impact point, due to the

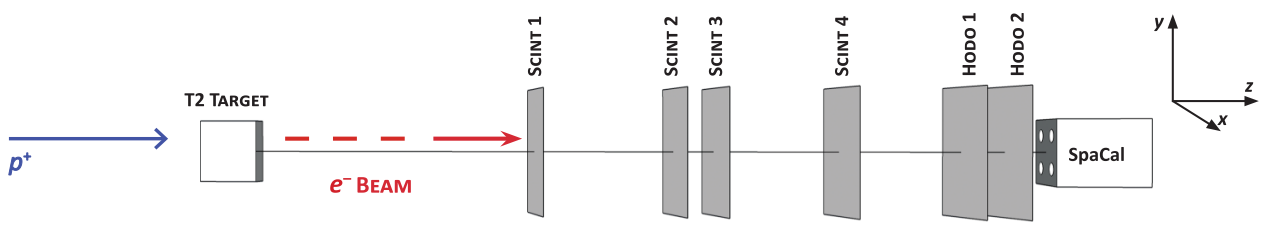

FIG. 2. The last stage of the H4 beam line at CERN SPS. The SpaCal prototype, the scintillation counters, and the hodoscopes used in the analysis are shown. The blue arrow represents the primary proton beam impacting on the target; the red arrow represents the electron-beam direction. 

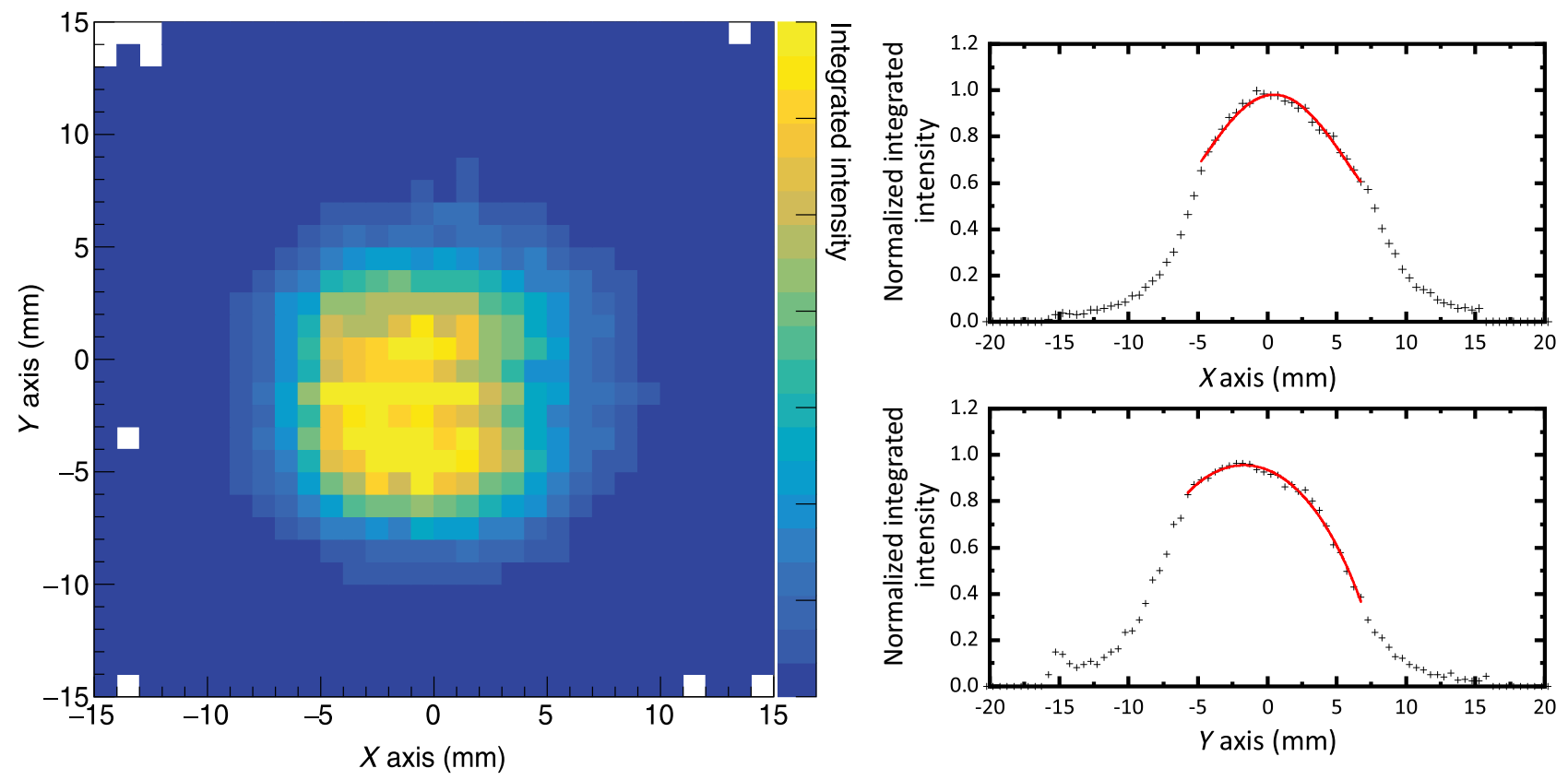

FIG. 3. The left panel shows a contour plot of the fiber average integrated intensity as a function of the impact point along $x$ and $y$ axes for a $150-\mathrm{GeV}$ electron beam with the module in the pointing configuration. A beam-spot selection of $20 \mathrm{~mm}^{2}$ is considered for the analysis. The right panels show projections of the average integrated intensity of a fiber channel as a function of the impact point along the $x$ axis (top-right panel) and the $y$ axis (bottom-right panel). Red lines represent a polynomial symmetric fit function $A\left(x-x_{0}\right)^{4}+B\left(x-x_{0}\right)^{2}+C$ used to determine the channel center.

variation of the shower containment and the consequent change of the energy deposited within the active volume: the center of each channel is defined as the position where the maximum response is observed, and this maximum is obtained by a fit to the measured response profiles with

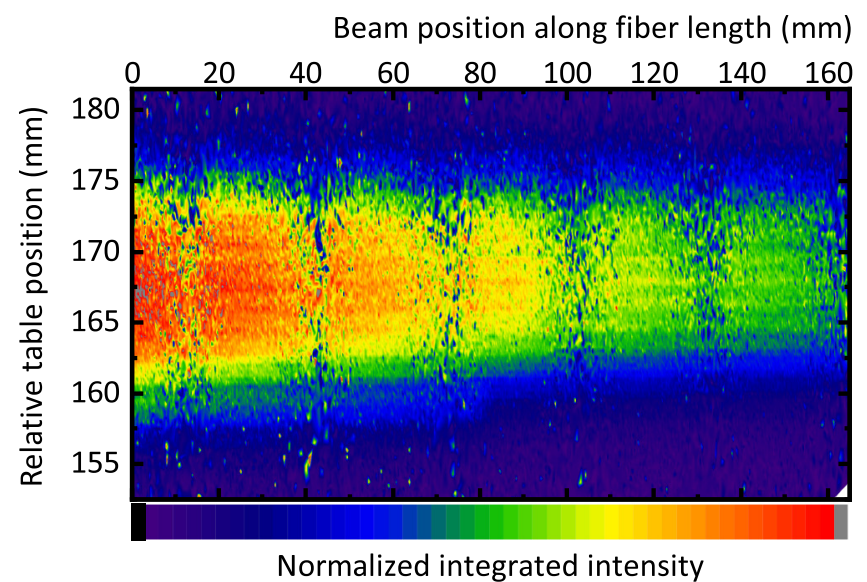

FIG. 4. Fiber signal in the transverse configuration as a function of beam position moving from left to right as detected by the left photodetector. On the $z$ axis, the integrated intensity normalized to the maximum reported from 0 to 1 on a linear scale is displayed. The vertical blue stripes are due to the small number of events at the edges of the beam profile when the table position is changed discretely in steps with respect to the beam. use of a fourth-order polynomial, as illustrated in the right panels in Fig. 3. This procedure allows the module to be correctly centered on the beam. The left panel in Fig. 3 shows a plot of the beam profile in the pointing configuration as detected by the rear PMT of the Ce-doped-fiber channel. Selection of a beam spot of $2.5-\mathrm{mm}$ radius from the center of the bundle, corresponding to an area of 20 $\mathrm{mm}^{2}$, is applied to limit shower transverse leakage.

After the position of each channel is determined, the high voltage supplied to each PMT is adjusted to have the highest signal without saturation: it is set to $1700 \mathrm{~V}$ for Cedoped silica fibers and $800 \mathrm{~V}$ for plastic fibers. This setting allows the use of the same PMT gain for the whole energy scan in the 20-200-GeV range.

Energy scans are performed with the beam incident on the center of each fiber bundle for 20-, 50-, 100-, 150-, and 200-GeV electrons.

As a last step, the whole box containing the module is rotated by $90^{\circ}$ clockwise to have the beam impacting on the lateral side of the fibers, allowing measurements of the attenuation length. A contour profile of the signal from Cedoped silica fibers in this latter configuration is shown in Fig. 4, as detected by the left PMT: it is the superposition of six different measurements obtained by shifting the detector in steps of $30 \mathrm{~mm}$ to allow the reconstruction of the attenuation profiles, further discussed in Sec. III D.

Before comparison of the signals from different channels, the photodetectors are intercalibrated in the 

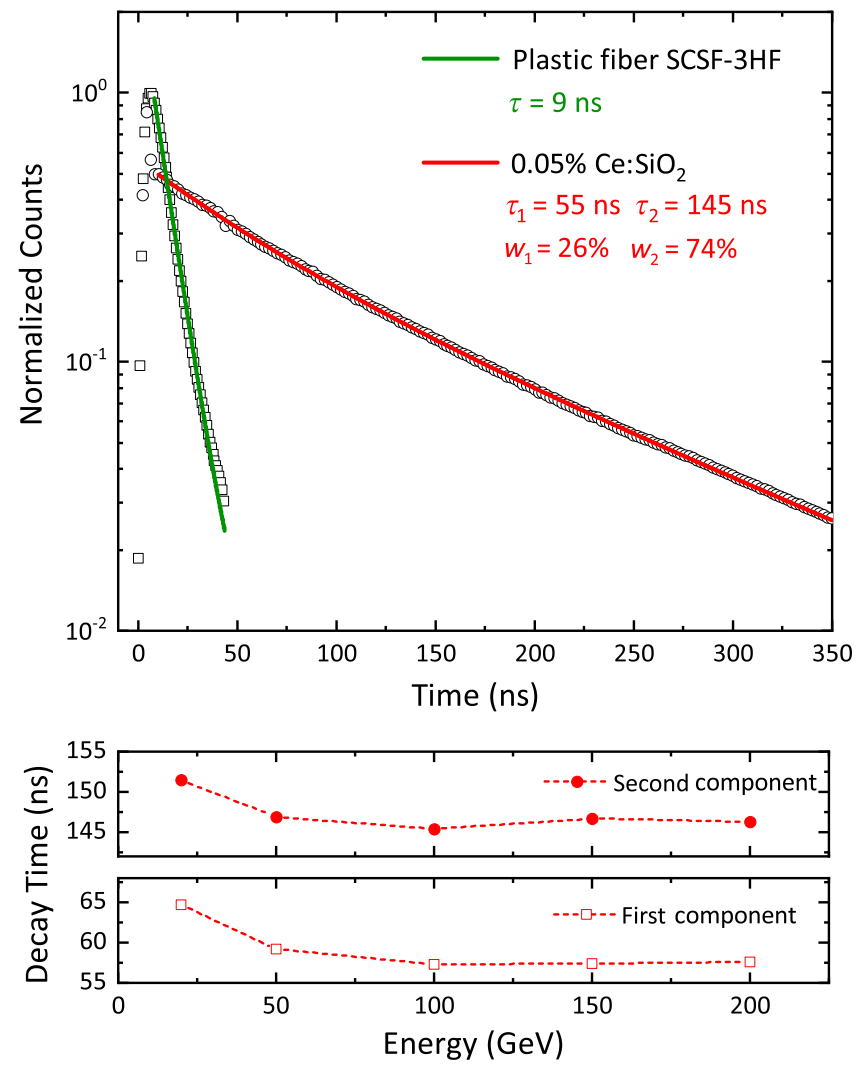

FIG. 5. The top panel shows the normalized average pulse shape of Ce-doped silica and plastic fibers (dotted lines) and a fit of the scintillation decay (solid lines) with a sum of exponential contributions for a $150-\mathrm{GeV}$ electron beam. The decay time and weight for each component are reported. The bottom panels show the decay time for the two components of scintillation decay of Ce-doped silica fibers as a function of the incident energy. The lines are guides for the eye.

laboratory. The intercalibration coefficients account for channel-by-channel response variations, due to the photocathode quantum efficiency and fiber-to-PMT optical coupling, and variations mostly related to the different high voltage applied to fit within the dynamic range of the analog-to-digital converter (ADC) and thus different amplification gain.

To estimate the amount of light produced in each fiber bundle, an absolute calibration of the photodetector response is also performed. A Ce: $\mathrm{Lu}_{2(1-x)} \mathrm{Y}_{2 x} \mathrm{SiO}_{5}$ crystal of $1 \mathrm{~mm}^{3}$ is used as a reference and measured under exposure to a ${ }^{137} \mathrm{Cs} \gamma$ source with each PMT used in the test-beam experiment: the reconstructed distribution is compared with that of a calibrated PMT to get the ADC-to-photoelectron conversion factors.

\section{TEST-BEAM RESULTS AND DISCUSSION}

The test-beam experiment and the following analysis provide a thorough investigation of the scintillation

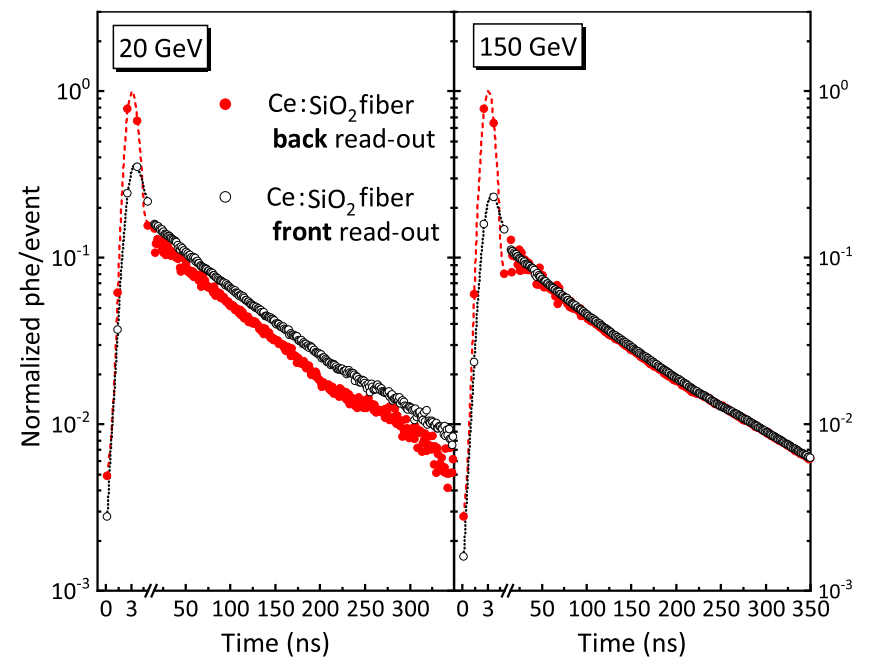

FIG. 6. Average pulse shape normalized to the number of events for $\mathrm{Ce}: \mathrm{SiO}_{2}$ fibers for a $20-\mathrm{GeV}$ electron beam (left panel) and a $150-\mathrm{GeV}$ electron beam (right panel). Back and front readout are compared. The dashed lines are guides for the eye. phe, photoelectrons.

properties of Ce-doped sol-gel silica fibers exposed to high-energy electromagnetic particles. The response of these fibers to high-energy electrons is studied with the SpaCal calorimetric module in both the pointing configuration and the transverse configuration, thus allowing evaluation of scintillation decay time, light output, and attenuation length.

The discussion focuses first on the pulse-shape analysis and then on the reconstruction of the shower profile and energy. A method for discrimination of Cherenkov and scintillation light is proposed to conduct a separate analysis of the two components of the emitted light, strengthening the possibility of Ce-doped silica fiber applications for simultaneous dual readout.

\section{A. Pulse-shape analysis}

The waveforms from the different types of fiber in the SpaCal module are recorded in a 350-ns time interval and reported in the top panel in Fig. 5: they are calculated by averaging over the selected events for $150-\mathrm{GeV}$ electrons having their impact point within $2.5-\mathrm{mm}$ radius from the center of a given channel, as described in Sec. II C.

We find that $0.002 \% \mathrm{Ce}: \mathrm{SiO}_{2}$ fibers do not show any scintillation light, probably too weak to be detected, and no analysis is performed on their luminescence decay.

Attention thus focuses on the comparison between the decays of $0.05 \% \mathrm{Ce}: \mathrm{SiO}_{2}$ and plastic fibers, which are fitted by the sum of exponential contributions. Plastic fiber scintillation decay is characterized by a single component with decay time $\tau=9 \pm 1 \mathrm{~ns}$, whereas Ce-doped silica fibers feature two contributions, with $\tau_{1}=55 \pm 5 \mathrm{~ns}$ and 

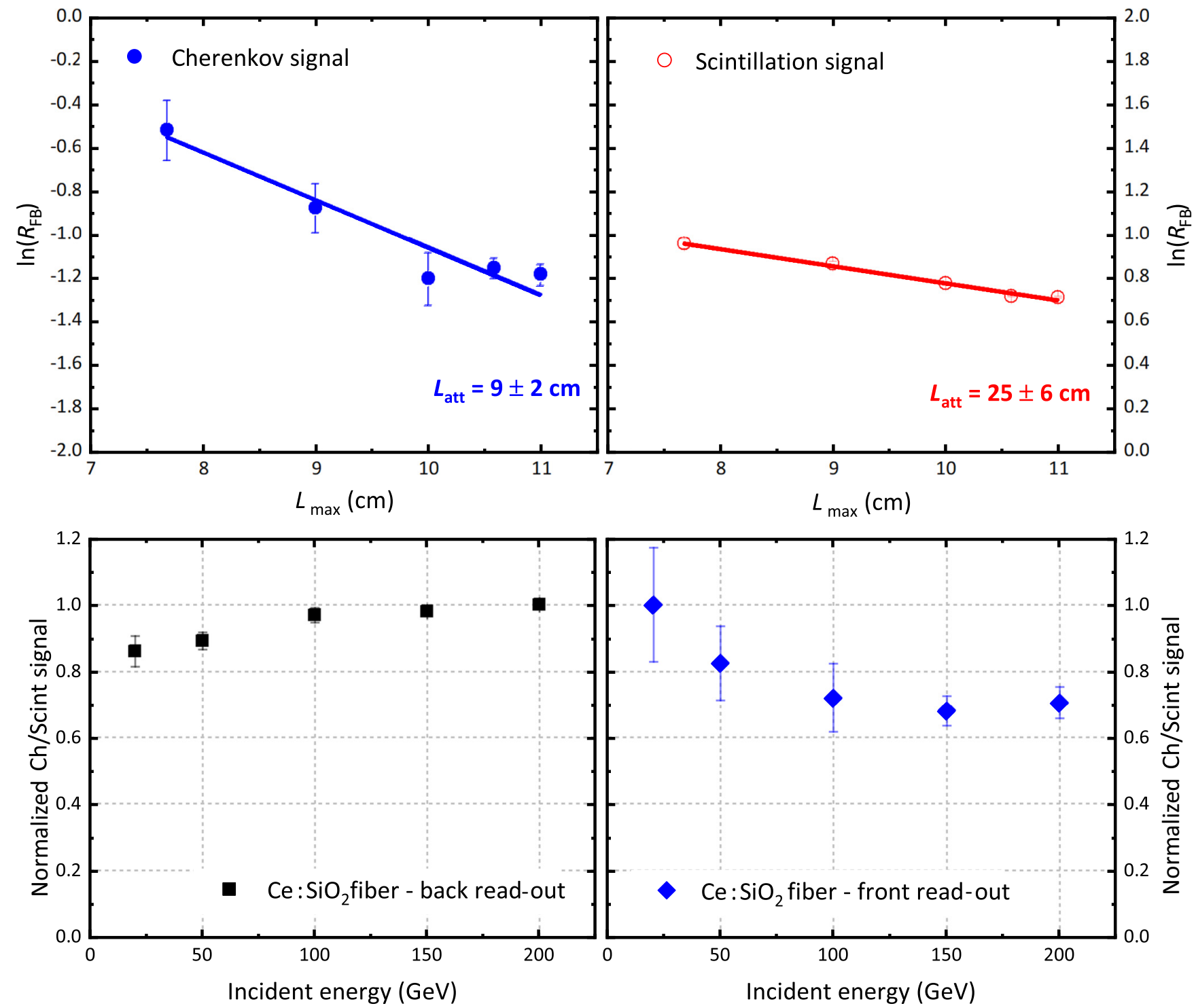

FIG. 7. The top panels show linear correlation between the natural logarithm of the ratio of front-PMT signal to the back-PMT signal and the position of the shower maximum in centimeters, proportional to the natural logarithm of the incident energy according to Eq. 1 for Cherenkov light (top-left panel) and scintillation light (top-right panel). The bottom panels show the ratio of Cherenkov (Ch) signal to scintillation (Scint) signal as a function of the incident energy for back readout (bottom-left panel) and front readout (bottom-right panel).

$\tau_{2}=145 \pm 22 \mathrm{~ns}$, and fractional weights of $(26 \pm 3) \%$ and $(74 \pm 3) \%$, respectively. The possible presence of a third, faster component cannot be inferred by this analysis, because the first part of the decay is strongly influenced by the fast Cherenkov emission. In the bottom panel in Fig. 5, the evolution of the decay time for the two components of Ce scintillation decay is reported as a function of incident-beam energy: an overall longer scintillation-decay time at the lowest energies can be observed for both components. We suggest that this effect can be ascribed, from a general point of view, to a variation of the excitation density in the material with decreasing incident energy, which consequently leads to differences in the scintillation recombination kinetics [34]. This phenomenon will be the subject of further investigation to deeply characterize and compare the scintillation kinetics of Ce-doped sol-gel silica fibers under various ionizing radiation sources with different distributions of excitation density in the material.

The decay times of Ce-doped fused-silica fibers produced by dissolution of the dopant in the glass matrix were reported by Akchurin et al. [26] and were evaluated to be $20.8 \pm 5.4 \mathrm{~ns}$ and $93.0 \pm 12.6 \mathrm{~ns}$ for $\tau_{1}$ and $\tau_{2}$, respectively, in a 200 -ns time window, but with similar fractional weights. The shorter decay times, compared 

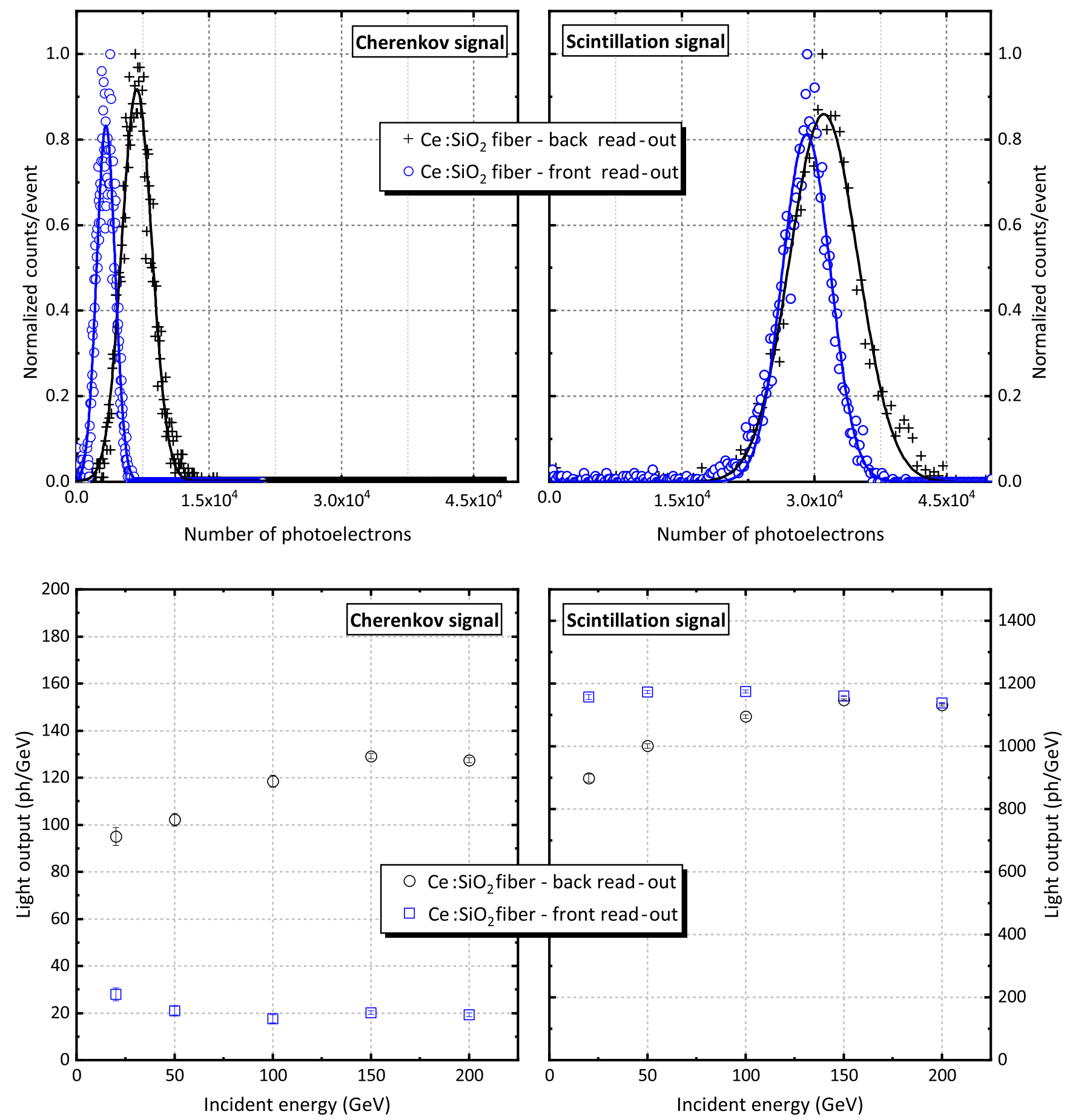

FIG. 8. The top panels show normalized distributions of photoelectrons for Cherenkov and scintillation signals for Ce: $\mathrm{SiO}_{2}$-fiber readout from both the ends for a $150-\mathrm{GeV}$ electron beam. The bottom panels show the correlation between light output of Cherenkov signal (bottom-left panel) and scintillation signal (bottom-right panel) and incident-beam energy. The $y$ axis reports the number of photons (ph) per $\mathrm{GeV}$ of incident energy, which is not therefore an absolute scale of the light yield of the material. Error bars are within the size of data points.

with those obtained in this study, can be ascribed to a different incorporation of Ce ions in the silica-based matrix studied in Ref. [26], containing a significant percentage of additional dopant ions, especially Al.

\section{B. Discrimination of Cherenkov and scintillation light for Ce-doped silica fibers}

In Fig. 6 the average pulse shape of Ce-doped silica fibers is displayed as recorded by the back PMT (red) and 
the front PMT (black) and normalized to the maximum of the back-PMT signal. The prompt emission, enlarged in the first part of the decay, can be ascribed to the Cherenkov effect [5,35-37], occurring in both the RE-doped core and the fluorinated cladding of glass fibers; the convolution with the scintillation emission has to be taken into account and, in the following, a detailed analysis is performed to distinguish the two contributions.

Cherenkov light is emitted in the forward direction at a characteristic angle with respect to the track of the charged particle. Thus, the Cherenkov signal is expected to be higher in the rear detector, confirmed by the amplitude of the peak in the first part of the waveform.

On the other hand, scintillation emission is isotropic, and the same amount of scintillation light is expected in both the rear detector and the front detector, as observed in the case of $150-\mathrm{GeV}$ incident electrons (Fig. 6, right panel). This occurs when the shower maximum falls approximately in the center of the fiber length, so the attenuation effects are the same for light traveling in the two directions. For the lowest energy of $20 \mathrm{GeV}$ (Fig. 6, left panel), the scintillation light in the front PMT is more intense than in the rear PMT, thus proving the dependence of the position of the maximum of the shower $L_{\max }$ on the incident-beam energy, described by Eq. 1 [38]:

$$
L_{\max }=X_{0}(4.2+1.8 \ln E),
$$

where $X_{0}=0.8 \mathrm{~cm}$ is the radiation length [32] of the calorimeter prototype evaluated by GEANT4 numerical simulation.

The average position of the shower maximum shifts toward the back of the absorber with increase of the energy of incoming electrons: this change in the behavior of the shower profile along the longitudinal axis is expected to produce a different ratio $R_{\mathrm{FB}}$ of the front-PMT signal ( $\left.S_{\text {front }}\right)$ and the back-PMT signal $\left(S_{\text {back }}\right)$ measured at the two opposite ends of the fibers, defined in Eq. 2:

$$
R_{\mathrm{FB}}=\frac{S_{\text {front }}}{S_{\text {back }}} .
$$

In the top panels in Fig. $7, R_{\mathrm{FB}}$ is plotted against the position of the maximum of the shower $\left(L_{\max }\right)$ in centimeters on a semilogarithmic scale. The average ratio $R_{\mathrm{FB}}$ of the front-PMT and back-PMT signal decreases due to the shift of the shower maximum toward the rear side of the absorber block at high energies. It is evaluated for both Cherenkov (left panel) and scintillation light (right panel), and a clear correlation with the beam energy can be observed and used for estimation of the attenuation length of the fibers with the calorimetric module in the pointing configuration, as discussed in Ref. [5]. The attenuation values so obtained are $L_{\text {att,Ch }}=9 \pm 2 \mathrm{~cm}$ and $L_{\text {att,scint }}=25 \pm 6 \mathrm{~cm}$ for Cherenkov and scintillation emission, respectively. The different nature of Cherenkov and scintillation light can account for the different correlations displayed in Fig. 7.

In the bottom panels in Fig. 7, the ratio of the Cherenkov signal to the scintillation signal as a function of beam energy is reported for the back readout (left) and the front readout (right) to evidence the different dependence of the two emissions on the shift of the shower maximum position toward the back of the module with increasing incident energy. The intensity of Cherenkov light increases in the back channel and decreases in the front channel faster than the intensity of scintillation, with the shower maximum moving toward the back, thus suggesting a greater attenuation of UV Cherenkov photons. This result is stressed in Sec. III D, where the attenuation length in the transverse configuration is evaluated for both emission contributions.

A separate study of Cherenkov and scintillation signals is performed through an event-by-event pulse-shape analysis, by integrating the waveform of the single selected event in different time intervals; namely, in the first $5 \mathrm{~ns}$ for Cherenkov emission, and in the remaining part for the scintillation contribution. This method leads to underestimation of the scintillation component because it does not take into account the scintillation signal emitted in the range $0<t<5 \mathrm{~ns}$, and thus a reconstruction of the true scintillation integrated emission has to be performed with use of the fit procedure conducted on the average waveform in Fig. 5.

By defining $S$ and $C$ as the integrated scintillation and Cherenkov emissions in the two separated time intervals calculated event by event, we can estimate the true integrated signals $S_{t}$ and $C_{t}$ by solving the following set of equations:

$$
\begin{aligned}
S_{t} & =\frac{S}{a}, \\
C_{t} & =C-S\left(\frac{1}{a}-1\right),
\end{aligned}
$$

where $a$ is the ratio between the scintillation integrated signal evaluated at $t>5 \mathrm{~ns}$ and the extrapolation to $t=0$ ns of the area under the exponential-decay-fit curve of the average pulse shape displayed in Fig. 5.

This method is used to reconstruct the distributions of photoelectrons for both Cherenkov and scintillation emission for the $150-\mathrm{GeV}$ electron beam, reported as an example in the top panels in Fig. 8, where the ADCto-photoelectron conversion factors are also taken into account. A difference of $3 \%-4 \%$ in the evaluated amount of scintillation signal between front and back readout can be observed in the top-right panel in Fig. 8: it is comparable to the precision of the calibration of the photodetectors. A Gaussian fit is performed on the reconstructed distributions in the 20-200-GeV energy range to estimate the peak positions. In the bottom panels in Fig. 8, the position of the peak, converted to photons and divided by the incident 

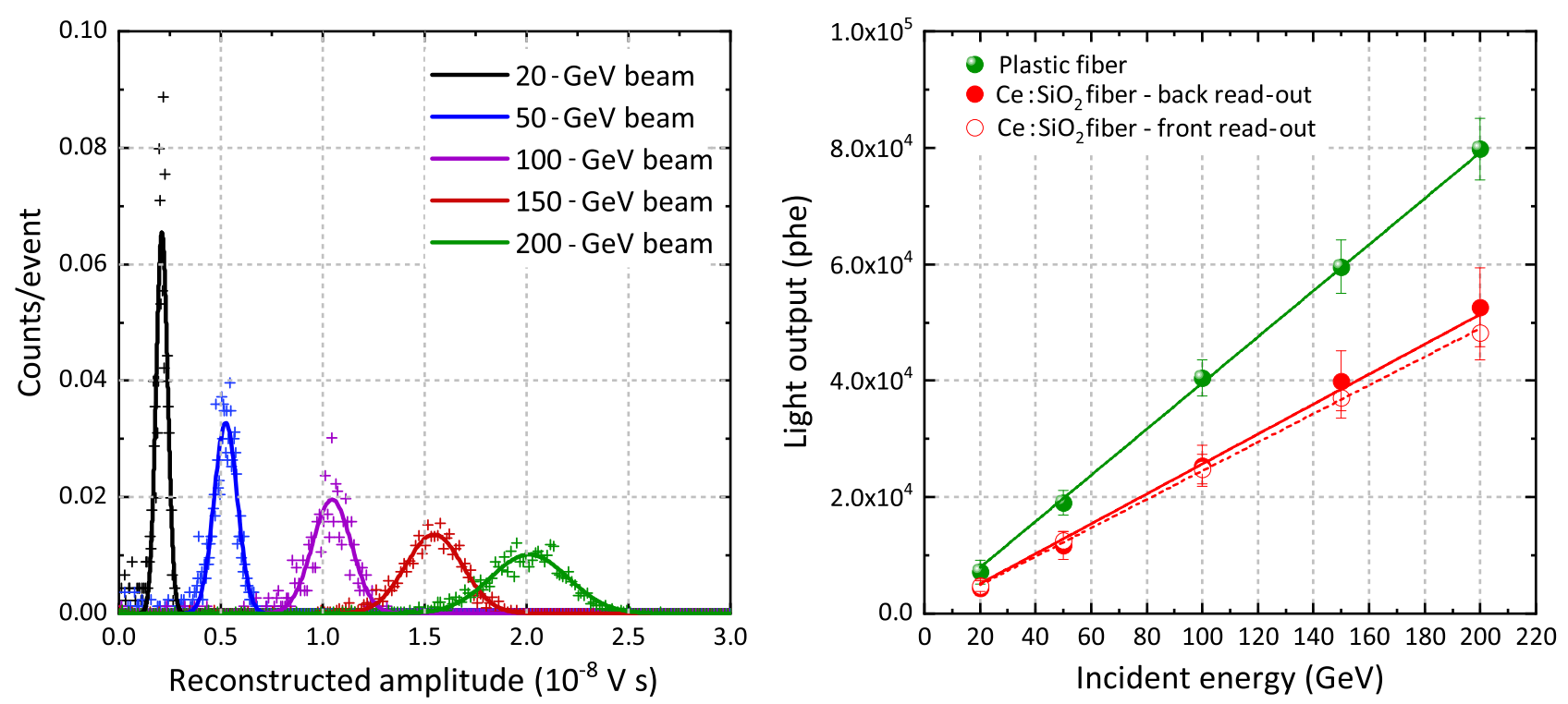

FIG. 9. The left panel shows distributions of reconstructed amplitude at different beam energies. Ce:SiO 2 -fiber signal is reported. The right panel shows linear correlation between peak values of reconstructed-amplitude distributions and incident-beam energy. The light output in photoelectrons (phe) is evaluated.

energy, is reported as a function of the incident energy itself: the light readout by the rear PMT shows a stronger nonproportionality with respect to the front readout. Such behavior can be related to the displacement of the maximum of the shower as a function of the incident energy, combined, only for the Cherenkov component, with the directionality of the emission. In any case, it can be noticed that the contribution of Cherenkov light is only around $5 \%-10 \%$ with respect to scintillation for all energies.

We can conclude that under exposure to a high-energyparticles-beam probe, the two contributions of Cherenkov and scintillation light can be detected simultaneously with the same Ce-doped silica fiber bundle. A characteristic dual response of the fibers is observed, and the feasibility to perform a well-distinguished analysis of the two components of the pulse shape is demonstrated.

\section{Energy reconstruction and linearity}

The particle energy can be reconstructed by application of intercalibration coefficients, calculated as described in Sec. IIC, and with consideration only of events within a beam spot of $2.5-\mathrm{mm}$ radius around the center of the fiber channel, to account only for electrons that develop the shower inside the fiber bundle. The total contribution is summed up event by event to reconstruct the signal, taking into account the ADC-to-photoelectron conversion factors. The distributions of the reconstructed amplitude obtained in the $20-200-\mathrm{GeV}$ range are shown in the left panel in Fig. 9 for Ce-doped silica fibers and fitted with a Gaussian function to estimate the peak position and its width.
The peak positions of the reconstructed amplitude distributions are converted to the number of photoelectrons and are reported in the right panel in Fig. 9, where good correlation with the incident-beam energy can be observed. A comparison of the light output of the different types of fibers is reported in the right panel in Fig. 9, showing that plastic fibers are brighter than Ce-doped silica fibers by a factor 1.5. It should be taken into account that the density of the plastic fibers $\left(1.2 \mathrm{~g} / \mathrm{cm}^{3}\right)$ is lower than that of silica fibers $\left(2.2 \mathrm{~g} / \mathrm{cm}^{3}\right)$, and therefore the incident beam deposits a lower fraction of energy in the plastic fiber channel.

However, the higher density of silica fibers can be regarded as an advantage because it permits to devise calorimeters with a shorter Molière radius compared with detectors that use plastic fibers as the active component; the shower is thus confined in a more-compact area.

The energy resolution $\sigma_{E} / E$ of the reconstructed amplitude is found to decrease with increasing incident energy, thus following the expected trend [39]: at $200 \mathrm{GeV}$ it is estimated to be around $15 \%$, which mainly accounts for contributions from the partial transverse shower containment within a single fiber bundle and from the nonuniformity of the fibers themselves.

\section{Attenuation length of Ce-doped silica fiber}

Nonuniformity along the fiber length in the efficiency of the light extraction at the fiber ends, if not corrected for (e g., by means of a double-sided readout [39]), can affect the response and the performance with high-energy particles. Characterization of the photoluminescence attenuation 

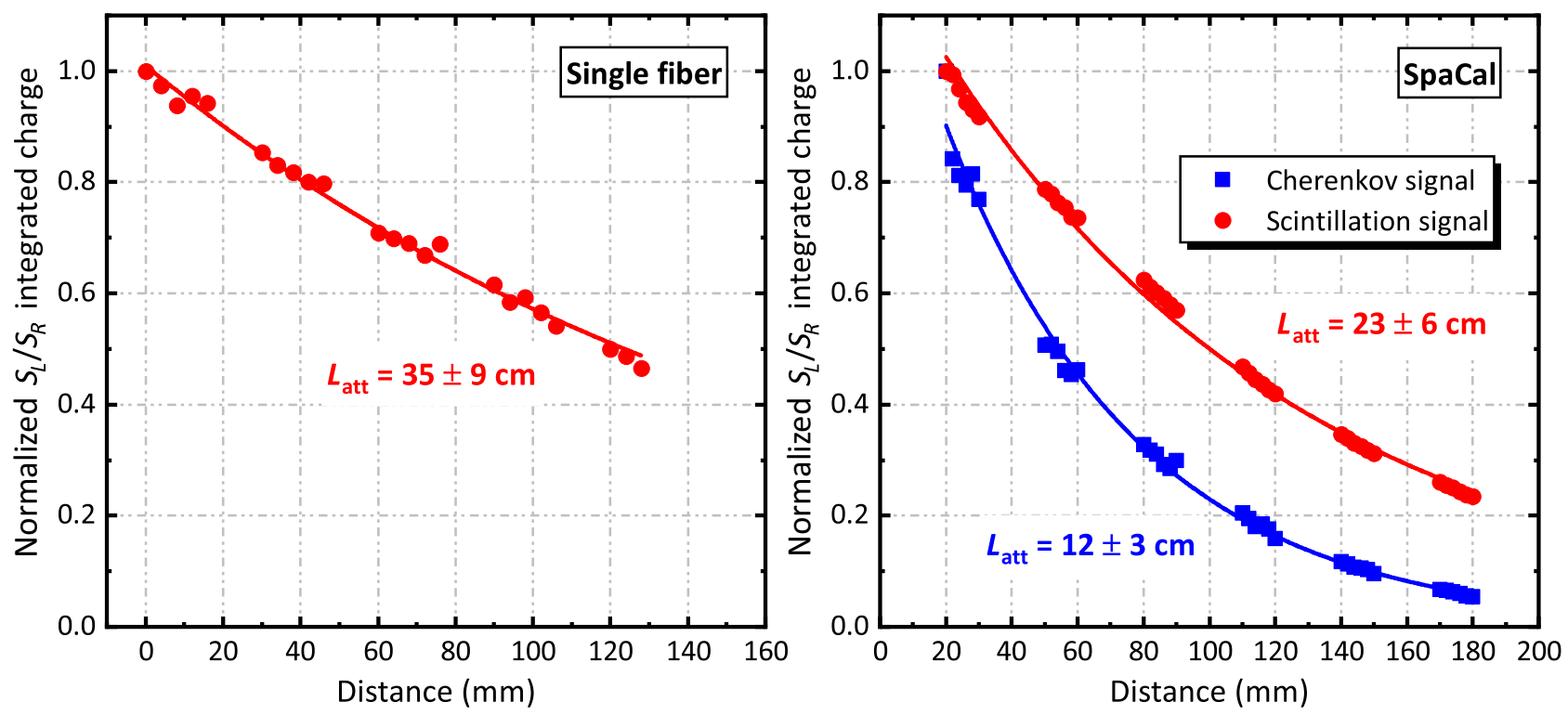

FIG. 10. Attenuation-length curves for $\mathrm{Ce}: \mathrm{SiO}_{2}$ fibers measured in the transverse configuration under exposure to a $150-\mathrm{GeV}$ electron beam and with use of the double-sided-readout technique: on the $y$ axis, the ratio of the left-PMT signal to the right-PMT signal is reported. The left panel shows measurements of a single fiber with UV-sensitive PMTs. The right panel shows attenuation measurements in the SpaCal configuration. Discrimination between Cherenkov signal and scintillation signal is considered.

length of Ce-doped silica fibers was previously conducted with a $370-\mathrm{nm}$ pulsed LED as the excitation source: the experimental procedure and results are described in Ref. [31].

The measurement is repeated with a $150-\mathrm{GeV}$ electron beam and the SpaCal module in the transverse configuration, with the table placed at six different $x$ coordinates to obtain a nearly uniform scan of the entire length of the fibers. To enhance the signal, a beam-spot selection of \pm 2 $\mathrm{mm}$ along the $y$ axis is applied for the computation of the energy deposited.

With use of the double-sided-readout technique, the transverse profiles are analyzed, parametrizing the curve with a single exponential decay according to Eq. 4:

$$
\frac{S_{L}}{S_{R}}=A_{0} \exp \left(-\frac{2 d}{L_{\text {att }}}\right),
$$

where $S_{L}$ and $S_{R}$ are the signals detected by the left PMT and the right PMT, respectively, $d$ is the distance, and $L_{\text {att }}$ is the characteristic attenuation length of the fiber.

Normalized transverse profiles and their fit are reported in Fig. 10: both single fibers (left panel) and fiber bundles in the SpaCal module (right panel) are measured, the former being read out with two UV-sensitive Hamamatsu Photonics H6610 PMTs, with use of two blocks of iron and lead as the preshower.

The discrimination of Cherenkov and scintillation signals is performed for the fibers in the SpaCal module, and the attenuation lengths estimated with the fit procedure are $L_{\mathrm{att}, \mathrm{Ch}}=12 \pm 3 \mathrm{~cm}$ and $L_{\text {att,scint }}=23 \pm 6 \mathrm{~cm}$ for Cherenkov and scintillation emission, respectively. Cherenkov light is observed to have a shorter attenuation length, thus confirming the results shown in Fig. 7 and described in Sec. III B: this could be ascribed to the fact that Cherenkov photons are mainly in the UV spectral region and have a higher probability therefore to be absorbed by Ce luminescent centers and by matrix defects.

For single fibers, only the analysis of the scintillation signal was possible due to the presence of spurious signal from the PMT windows. Scintillation attenuation length was found to be shorter in the SpaCal configuration with respect to single fibers $\left(L_{\mathrm{att}, \mathrm{scint}}=35 \pm 9 \mathrm{~cm}\right)$ : this could be related to the silica cladding in contact with the absorber, leading to a variation of the refractive index at the fiber boundary and to a loss in the total-internal-reflection efficiency, which causes an increase of light leakage before light reaches the photodetector.

Further optimization of the fiber cladding geometry and proper quenching of the cladding light-propagation modes are thus suggested by this analysis to improve the light extraction through total internal reflection. Nevertheless, the attenuation profile measured in the beam test for the single Ce-doped silica fiber is in good agreement with laboratory measurements [31].

\section{CONCLUSIONS}

Our results obtained under exposure to high-energy electrons allow a detailed investigation of the scintillation properties of Ce-doped sol-gel silica fibers embedded as the active component in a spaghetti-like electromagnetic 
calorimeter prototype. The decay time of $\mathrm{Ce}^{3+}$ scintillation, silica-fiber attenuation length, and relative light output are evaluated by our testing the SpaCal module in both the pointing configuration and the transverse configuration with respect to the incident beam. The scintillation time decay is analyzed and the reconstruction of the shower profile and energy are addressed.

The feasibility of a simultaneous dual-readout approach and the discrimination of Cherenkov and scintillation emitted light are demonstrated. This unique feature allows the realization of calorimeters made of a single type of fiber to read the dual emission instead of two types of fibers with either different doping or different density.

The results presented support the potential applications and perspectives of silica fibers for dual-readout calorimetry, as well as for beam monitoring, radiotherapy in the medical field, security, and industrial x-ray imaging.

Further material engineering will aim for optimization of the fiber geometry toward complete quenching of the cladding propagation modes to improve the light extraction from the fiber core together with the radiation hardness to exploit the possibility that sol-gel silica offers to realize long RE-doped or undoped silica fibers with good attenuation length in a cost-effective way.

\section{ACKNOWLEDGMENTS}

The authors sincerely thank Andrea Polesel, Rosalinde Pots, Nazar Bartosik, Alessandra Cappati, and Muhammad Bilal Kiani for taking part in the test-beam campaign and for all the time and work they put into it. The authors are grateful also to Hubert Gerwig and Nicolas Siegrist from CERN EP-CMX for the realization of the SpaCal absorber. This work was conducted in the frame of the Crystal Clear collaboration and was supported by the Horizon 2020 RISE Intelum project (Grant No. 644260), the Horizon 2020 Research Infrastructures (AIDA-2020) (Grant No. 654168), the ASCIMAT project (Grant No. 690599), and the EIT SPARK project (Grant No. 16290).

[1] I. Veronese, C. De Mattia, M. Fasoli, N. Chiodini, E. Mones, M. C. Cantone, and A. Vedda, Infrared luminescence for real time ionizing radiation detection, Appl. Phys. Lett. 105, 061103 (2014).

[2] N. Savard, D. Potkins, J. Beaudry, A. Jirasek, C. Duzenli, and C. Hoehr, Characteristics of a Ce-doped silica fiber irradiated by $74 \mathrm{MeV}$ protons, Radiat. Meas. 114, 19 (2018).

[3] P. Lecoq, New crystal technologies for novel calorimeter concepts, J. Phys. Conf. Ser. 160, 012016 (2009).

[4] C. Dujardin, C. Mancini, D. Amans, G. Ledoux, D. Abler, E. Auffray, P. Lecoq, D. Perrodin, A. Petrosyan, and K. L. Ovanesyan, LuAG:Ce fibers for high energy calorimetry, J. Appl. Phys. 108, 013510 (2010).
[5] M. Lucchini, T. Medvedeva, K. Pauwels, C. Tully, A. Heering, C. Dujardin, K. Lebbou, P. Lecoq, and E. Auffray, Test beam results with LuAG fibers for next-generation calorimeters, J. Instrum. 8, P10017 (2013).

[6] A. Benaglia, M. Lucchini, K. Pauwels, C. Tully, T. Medvedeva, A. Heering, C. Dujardin, V. Kononets, K. Lebbou, N. Aubry, S. Faraj, G. Ferro, P. Lecoq, and E. Auffray, Test beam results of a high granularity LuAG fibre calorimeter prototype, J. Instrum. 11, P05004 (2016).

[7] K. Pauwels, M. Lucchini, A. Benaglia, and E. Auffray, in Engineering of Scintillation Materials and Radiation Technologies, edited by M. Korzhik and A. Gektin (Springer International Publishing, Cham, 2017), p. 231.

[8] R. Becker, G. Dissertori, A. Gendotti, Q. Huang, D. Luckey, W. Lustermann, S. Lutterer, F. Nessi-Tedaldi, F. Pandolfi, and F. Pauss, Proof-of-principle of a new geometry for sampling calorimetry using inorganic scintillator plates, J. Phys. Conf. Ser. 587, 012039 (2015).

[9] D. Contardo, M. Klute, J. Mans, L. Silvestris, and J. Butler, Tech. Rep. No. CERN-LHCC-2015-010. LHCC-P008.CMS-TDR-15-02 (CERN, Geneva, 2015).

[10] S. Lee, M. Livan, and R. Wigmans, Dual-readout calorimetry, Rev. Mod. Phys. 90, 025002 (2017).

[11] N. Akchurin, O. Atramentov, K. Carrell, K. Z. Gümüs, J. Hauptman, H. Kim, H. P. Paar, A. Penzo, and R. Wigmans, Separation of scintillation and Cherenkov light in an optical calorimeter, Nucl. Instrum. Methods Phys. Res. A 550, 185 (2005).

[12] E. Auffray, D. Abler, P. Lecoq, and G. Mavromanolakis, Dual readout with PWO crystals and LuAG crystal scintillating fibers, IEEE Trans. Nucl. Sci. 57, 1454 (2010).

[13] N. Akchurin et al., Detection of electron showers in dualreadout crystal calorimeters, Nucl. Instrum. Methods Phys. Res. A 686, 125 (2012).

[14] W. J. Yoo, S. H. Shin, D. Jeon, S. Hong, S. G. Kim, H. I. Sim, K. W. Jang, S. Cho, and B. Lee, Simultaneous measurements of pure scintillation and Cerenkov signals in an integrated fiber-optic dosimeter for electron beam therapy dosimetry, Opt. Express 21, 27770 (2013).

[15] T. M. Shaffer, C. M. Drain, and J. Grimm, Optical imaging of ionizing radiation from clinical sources, J. Nuc. Med. 57, 1661 (2016).

[16] A. Arodzero, S. Boucher, J. Hartzell, S. V. Kutsaev, R. C. Lanza, V. Palermo, S. Vinogradov, and V. Ziskin, in Proc. IEEE Nucl. Sci. Symp. and Med. Imaging Conf. (NSS/MIC) (2015), p. 1.

[17] A. Arodzero, U.S. Patent No. 2011/0163236, (2011).

[18] M. Christl, J. H. Adams, Jr., E. N. Kuznetsov, and S. Nazzel, in PROC. OF THE 31st ICRC (Local Organizing Committee of ICRC, Lodz, Poland, 2009).

[19] M. T. Lucchini, K. Pauwels, K. Blavzek, S. Ochesanu, and E. Auffray, Radiation tolerance of LuAG:Ce and YAG:Ce crystals under high levels of gamma- and proton-irradiation, IEEE Trans. Nucl. Sci. 63, 586 (2016).

[20] B. J. Singleton, B. S. Jones, A. A. Bickley, J. C. Petrosky, J. W. McClory, and B. R. Kowash, in IEEE Nucl. Sci. Symp. Conf. Record (IEEE, Valencia, Spain, 2011), p. 1935. 
[21] K. Pauwels, C. Dujardin, S. Gundacker, K. Lebbou, P. Lecoq, M. Lucchini, F. Moretti, A. G. Petrosyan, X. Xu, and E. Auffray, Single crystalline LuAG fibers for homogeneous dual-readout calorimeters, J. Instrum. 8, P09019 (2013).

[22] V. Kononets, E. Auffray, C. Dujardin, S. Gridin, F. Moretti, G. Patton, K. Pauwels, O. Sidletskiy, X. Xu, and K. Lebbou, Growth of long undoped and Ce-doped LuAG single crystal fibers for dual readout calorimetry, J. Cryst. Growth 435, 31 (2016).

[23] A. E. Dosovitskiy, G. A. Dosovitskiy, and M. V. Korjik, in PROC. SPIE (San Diego, USA, 2012).

[24] Z. Kang, B. K. Wagner, C. J. Summers, J. Nadler, R. Rosson, and B. Kahn, in IEEE Nuclear Science Symposium and Medical Imaging Conference (NSS/MIC) (IEEE, Anaheim, USA, 2012), p. 1688.

[25] T. Yanagida, J. Ueda, H. Masai, Y. Fujimoto, and S. Tanabe, Optical and scintillation properties of Ce-doped $34 \mathrm{Li}_{2} \mathrm{O}-5 \mathrm{MgO}-10 \mathrm{Al}_{2} \mathrm{O}_{3}-51 \mathrm{SiO}_{2}$ glass, J. Non-Cryst. Solids 431, 140 (2016).

[26] N. Akchurin, C. Cowden, J. Damgov, C. Dragoiu, P. Dudero, J. Faulkner, and S. Kunori, Cerium-doped scintillating fused-silica fibers, J. Instrum. 13, P04010 (2018).

[27] A. Vedda, N. Chiodini, D. Di Martino, M. Fasoli, S. Keffer, A. Lauria, M. Martini, F. Moretti, and G. Spinolo, $\mathrm{Ce}^{3+}$ doped fibers for remote radiation dosimetry, Appl. Phys. Lett. 85, 6356 (2004).

[28] A. Vedda, N. Chiodini, D. Di Martino, M. Fasoli, F. Morazzoni, F. Moretti, R. Scotti, G. Spinolo, A. Baraldi, R. Capelletti, M. Mazzera, and M. Nikl, Insights into microstructural features governing $\mathrm{Ce}^{3+}$ luminescence efficiency in sol-gel silica glasses, Chem. Mater. 18, 6178 (2006).

[29] A. Penzo, Y. Onel, and the CMS Collaboration, The CMSHF quartz fiber calorimeters, J. Phys.: Conf. Ser. 160, 012014 (2009).
[30] F. Cova, M. Fasoli, F. Moretti, N. Chiodini, K. Pauwels, E. Auffray, M. T. Lucchini, E. Bourret, I. Veronese, E. d'Ippolito, and A. Vedda, Optical properties and radiation hardness of Pr-doped sol-gel silica: Influence of fiber drawing process, J. Lumin. 192, 661 (2017).

[31] F. Cova, F. Moretti, M. Fasoli, N. Chiodini, K. Pauwels, E. Auffray, M. T. Lucchini, S. Baccaro, A. Cemmi, H. Bártová, and A. Vedda, Radiation hardness of Ce-doped sol-gel silica fibers for high energy physics applications, Opt. Lett. 43, 903 (2018).

[32] P. A. Rodnyi, Physical processes in inorganic scintillators, (CRC Press, Boca Raton, US, 1997), Vol. 14

[33] J. Spanggaard, Delay Wire Chambers - A Users Guide, Tech. Rep. No. SL-Note-98-023-BI (CERN, Geneva, 1998).

[34] A. N. Vasil'ev, in International Conference on Engineering of Scintillation Materials and Radiation Technologies (Springer, Minks, Belarus, 2016), p. 3.

[35] C. Grupen and B. Shwartz, Particle detectors (Cambridge University Press, New York, US, 2008).

[36] P. Krizan, Recent progress in particle identification methods, Nucl. Instrum. Methods Phys. Res. A 598, 130 (2009).

[37] J. P. Hayward, C. L. Hobbs, Z. W. Bell, L. A. Boatner, R. E. Johnson, J. O. Ramey, G. E. Jellison, and C. R. Lillard, Characterizing the radiation response of Cherenkov glass detectors with isotopic sources, J. Radioanal. Nucl. Chem. 295, 1143 (2013).

[38] C. W. Fabjan and F. Gianotti, Calorimetry for particle physics, Rev. Mod. Phys. 75, 1243 (2003).

[39] M. T. Lucchini, E. Auffray, A. Benaglia, F. Cavallari, D. Cockerill, A. Dolgopolov, J. L. Faure, N. Golubev, P. R. Hobson, S. Jain, M. Korjik, V. Mechinski, A. Singovski, T. Tabarelli de Fatis, I. Tarasov, and S. Zahid, Double side readout technique for mitigation of radiation damage effects in $\mathrm{PbWO}_{4}$ crystals, J. Instrum. 11, P04021 (2016). 